\title{
Providing a New Model for Discovering Cloud Services Based on Ontology
}

\author{
Bahram Heydari \\ Department of Computer Engineering \\ Zanjan Branch, Islamic Azad University \\ Zanjan, Iran \\ bh.heydari2016@gmail.com
}

\author{
Mojtaba Aajami \\ Department of Computer Engineering \\ Zanjan Branch, Islamic Azad University \\ Zanjan, Iran \\ m.aajami@gmail.com
}

\begin{abstract}
Due to its efficient, flexible, and dynamic substructure in information technology and service quality parameters estimation, cloud computing has become one of the most important issues in computer world. Discovering cloud services has been posed as a fundamental issue in reaching out high efficiency. In order to do one's own operations in cloud space, any user needs to request several various services either simultaneously or according to a working routine. These services can be presented by different cloud producers or different decision-making policies. Therefore, service management is one of the important and challenging issues in cloud computing. With the advent of semantic web and practical services accordingly in cloud computing space, access to different kinds of applications has become possible. Ontology is the core of semantic web and can be used to ease the process of discovering services. A new model based on ontology has been proposed in this paper. The results indicate that the proposed model has explored cloud services based on user search results in lesser time compared to other models.
\end{abstract}

Keywords-cloud; computing; service; semantic; web; ontology

\section{INTRODUCTION}

Cloud computing includes a lot of remote servers which make a network and cause focused data storage and online access to services and computer sources [1]. To put it in another way, cloud computing is access to calculation sources through the internet and in effect instead of saving your data on the hard disc or updating your practical applications constantly, you can use an internet service to meet such needs [2]. Cloud computing is a calculating pattern in which numerous systems are linked in the form of private or public networks to make a dynamic and comparable substructure for practical applications for the purpose of saving data and files [3]. With the emergence of this technology, hosting practical applications, saving content and delivering services has reduced remarkably. The idea of cloud computing in fact is on the basis of "Reusing Technology Capabilities". Ontology is a kind of structured knowledge about a specific area which is made by presenting concepts and relations among them [4]. Nowadays, because of designing ontology in different fields, offering criteria for the selection of the most appropriate ontology is essential. Ontology is a branch of philosophy which studies essence of existence and structure of reality, but, in semantic web space, ontology investigates the classification of topics or the elements in a specific field and offers a list of them. Few studies have been conducted on cloud service discovery $[5,6]$ all of which have aimed to upgrade the quality of exploration process and combine cloud services from various perspectives. With the improvement of communicational substructures and educational practices, the use of cloud computing has increased. Given the widening use of the internet, cloud services have become a major data tool on the internet. The first major problem in exploring cloud services is the necessity of identification, exploration, and optimal selection from among a wide range of services. Hence, the more accurate the process of cloud service discovery, the more efficient the system functioning. The second problem is the lack of access to the exploration of the users' favorite services. In the majority of cases, cloud service is not able to explore the best service. As a result, ontology is a new method for exploring co-related services based on semantic relations.

A lot of companies offer cloud computing services. Cloud space is a service for offering storage space to the users so that they can save their information on the internet in order to have access to them from anywhere or by different devices [7]. Cloud computing makes it possible for users and developers to use them without getting involved with technical information or control of the needed technological substructures. One of the major issues in cloud computing is service exploration. Sharing, source allocation, purification and service exploration are of great prominence while data and software are offered in the form of service in cloud computing. Under-exploration of appropriate services for the various user needs is the main problem in using cloud computing services. With the emergence of semantic web and semantic web services after that, exploring cloud web service has gained crucial importance. Ontology is the core of semantic web and can be used to ease the process of exploring web services [8]. The aim of exploring web services is to search and find the appropriate web services which meet the users' needs.

\section{RELATED WORKS}

The aim of developing semantic web services is to use the technological capabilities of semantic web to auto-explore, 
combine, monitor, and run them. Few studies have been done in this regard, and different solutions have been proposed to explore cloud service. Authors in [9] proposed a multi-factor ontology-based system to explore cloud service. A multi-factor system is composed of several interactional intelligent factors. Multi-factor systems can be used to solve problems, the solution of which is difficult or impossible for a pure system. Multi-factor systems have better efficiency in searching, parallel, or algorithmic processing. In the hybrid model, the semantic relations between services are based on ontology being explored based on a multi-factor system. A model has been proposed on the basis of ontological clustering to explore cloud substructure services [10]. Substructure-based clusters have been created as a service and the ontology of the semantic relations among them has been established. Calculation sources have been considered as the provider of the service and storage space in ontological clustering. The results indicate that the clustering model has explored cloud substructure services in less time than the normal method. Authors in [11] proposed a hybrid model based on ant colony optimization and learning automaton to explore the cloud semantic relations. Ant colony optimization has been used to find the shortest paths and reach the intended service in the hybrid model. Learning automaton has been used to improve ant colony optimization algorithm and to make it intelligent. The results indicate that the hybrid model obtains better outcomes in less time.

A model has been proposed based on XML substructure for cloud service discovery [12]. It aims to provide cloud services based on XML files. In this model, XML file consists of 3 parts including memory, processor, and cloud space services. Each part in XML files is labeled and the users can select their favorite service. Authors in [13] proposed a model based on semantic web for educational spaces in cloud computing. Since search engines are used to internet search and they show information unrelated to the search, the data searched are only recognized by the motor engine and they cannot be interpreted by the motor engines. Consequently, semantic web model which is used as the new technology has a major impact on cloud education services and ontology investigation in the retrieval and extraction of data. Ontology describes different aspects of the learning process in cloud space. Specifically, the manager of a database considers the cloud knowledge as the core of learning which instructs the learner to find the educational services in cloud with the help of ontology. Data production and maintenance in cloud knowledge dataset is managed by ontological techniques. Authors in [14] proposed a new model based on argumentation factors for cloud service discovery based on ontology. Word frequency has been used to relate the data in this model. The data whith higher frequency and closer semantic relation for service exploration is retrieved. SAAS, PAAS, and IAAS interact based on ontology and the best service is retrieved.

\section{THE PROPOSED MODEL}

Ontology is the structured study of a specific field which is formed by proposing the concepts and the relations among them. In the proposed model, the relations among the cloud services are explored based on ontology, and the best services are selected for a specific function. Cloud-based services are done at SAAS, PAAS, and IAAS levels. In the first level, the user inserts his/her request. According to the user's request, the process is done in a XML file. In the proposed architecture, the task of XML dataset is to store a mass of services, return the results intended by the users as soon as possible and in some cases process some of the saved services. As the data are heterogeneous and complex in XML, inquiries are made to enhance the quality of retrieval. RDF can be used to develop inquiries. In the proposed model, the services requested by the user are sent to the cloud provider based on ontology, and the cloud provider must provide the sources needed based on the wanted services. The flowchart of the proposed model is shown in Figure 1. The most important services for the users lie in IAAS level. This level includes such services as storage space, memory, and central processing module. Based on user operation, he/she needs memory and a processing system. Therefore, service selection must be done in a way that the most optimal and the most appropriate services get selected. By selecting the services on behalf of the users, cloud providers can offer the certificate needed so that users can have access to cloud space.

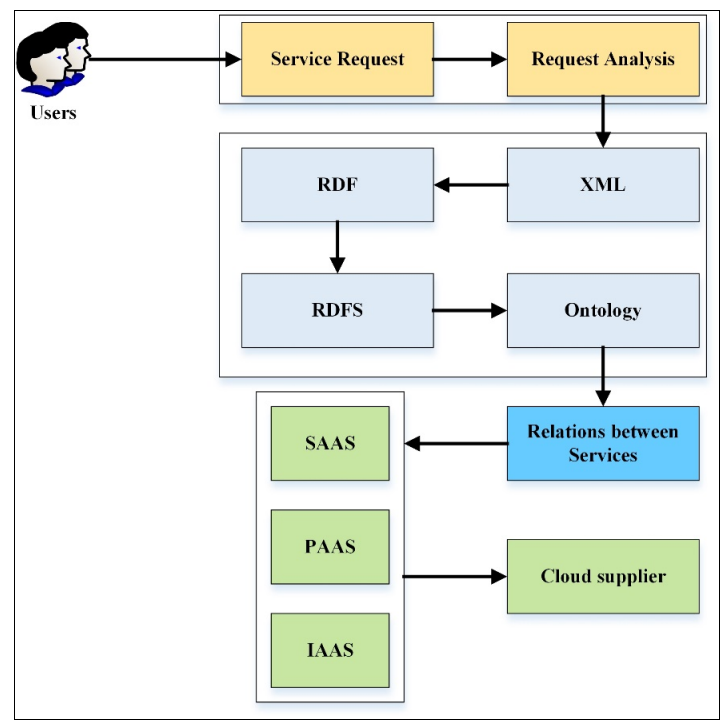

Fig. 1. The Proposed Model for Service Exploration Based on Ontology

\section{EVALUATION AND RESULTS}

Using SPARQL, modelling the proposed architecture was carried out on Protégé 5.0.0 software. Protégé is a software used in semantic web. The major task of this software is to produce content which suits the semantic web and ontology. Furthermore, there is the likelihood of retrieving ontology, developing it, making inquiries, graphical presentation of the classes, their identities, and the relations among them. 15 cloud providers and 150 different services have been used in the proposed architecture. The simulation scenario is shown in Figure 2. Ontology of the proposed architecture for cloud service discovery is shown in Figure 3 based on OntoGraf chart. Ontology of the cloud services is shown in Figure 4 based on OntoGraf chart. Each cloud service has practical subclasses which are used to explore services and build 
ontology relations. Ontology model of the cloud services is shown in Figure 5 based on OWLViz chart. Ontology model of cloud services is shown in Figure 6 based on OWLViz chart.

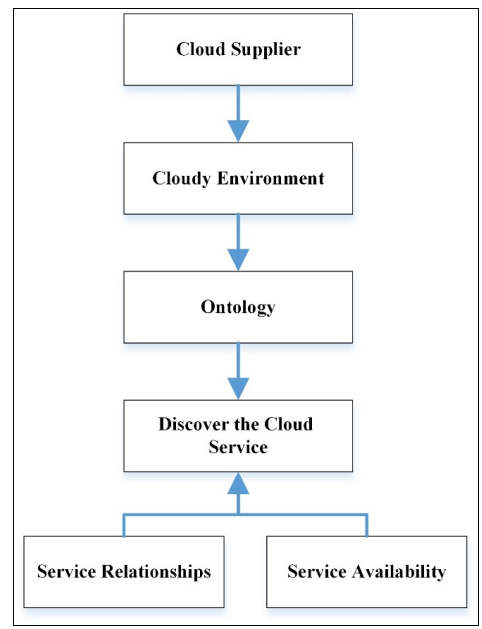

Fig. 2. Simulation Scenario

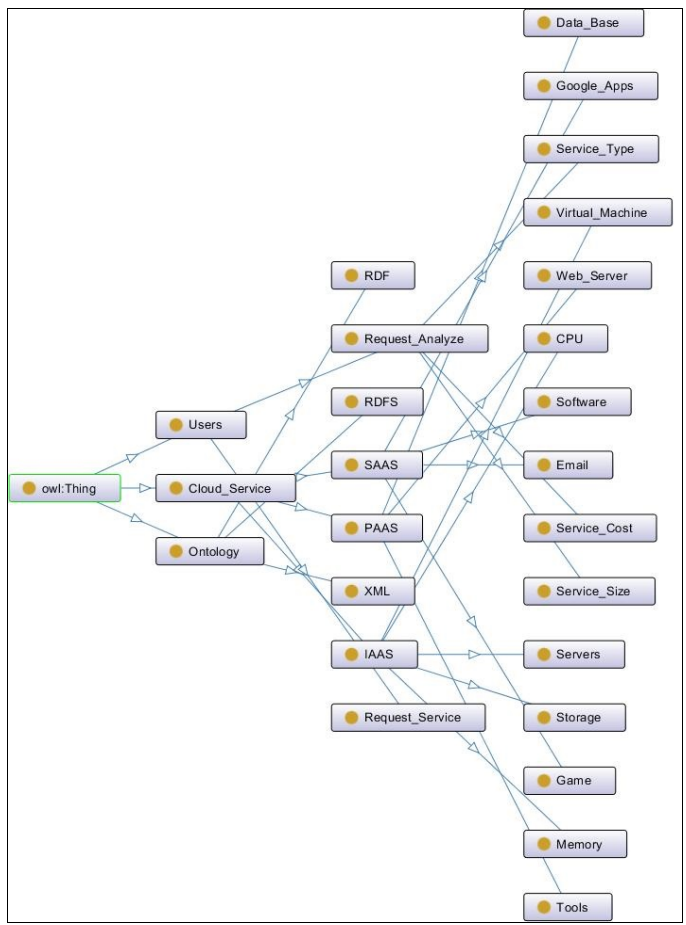

Fig. 3. Ontology of the Proposed Architecture for cloud service discovery on the basis of OntoGraf Chart

Inquiries (1), (2), (3), (4), (5), and (6) are examples of SPRALQL instructions which are written in Protégé space to explore cloud services based on the proposed model (Table I). In instruction (1), cloud service discovery is done based on SAAS. In (2), cloud service discovery is done based on PAAS. In (3), cloud service discovery is done based on IAAS. In (4), service exploration is done based on SAAS, PAAS, and IAAS. In (5), explored services are classifies based on IAAS. Services are shown based on numbers in classification. In instruction
(6), filtering operation is done on the explored services. In instruction (6), services are saved in the memory for the purpose of retrieval. A comparison of the search time is made for the exploration of services in Table II based on the number of providers and services. The search time period is different based on the number of providers and services. If the number of providers is high, the time period for searching will be more. This is because the search for exploring services is made among greater number of providers.

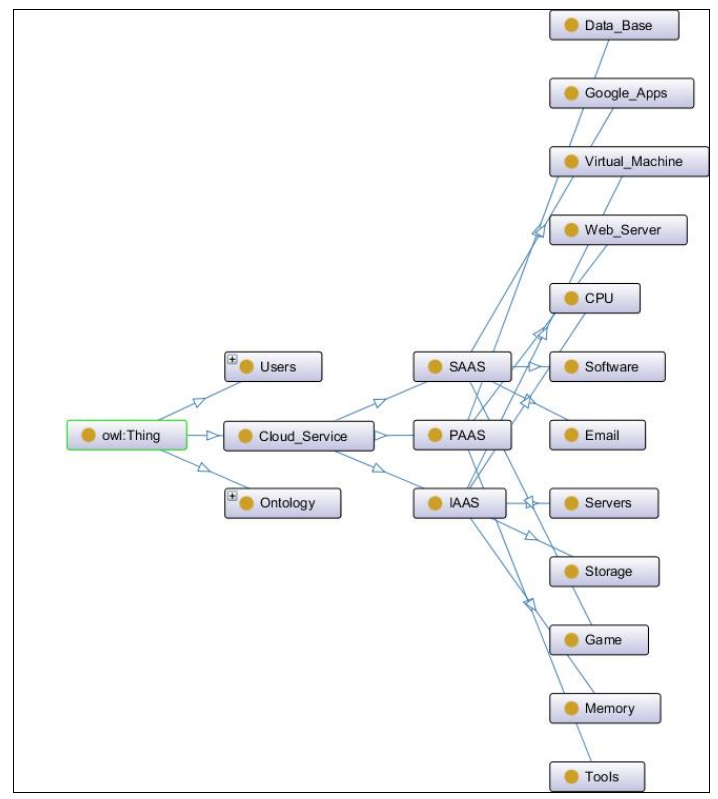

Fig. 4. Ontology Model of the Cloud Services based on OntoGraf Chart

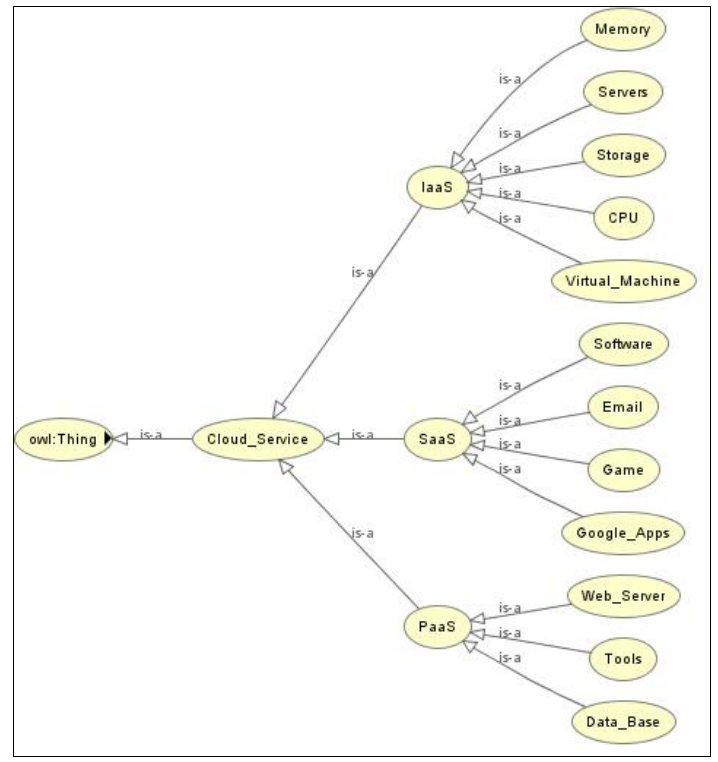

Fig. 5. Ontology Model of the Cloud Services based on OWLViz Chart 


\section{A. Comparison and Evaluation}

The main advantage of the proposed model is the reducing of the search time for service discovery. In the proposed model, based on ontology, services are discovered at a faster time. The search time for service discovery is very important for users, so service discovery should be done in the shortest possible time and with the best response time. Figure 7 shows the comparison of service discovery time of proposed model with others. The comparison is shown on the basis of 20 suppliers and 194 services. The search time for the discovery of the service in the cluster-free clustering model is 10152 and 5021 milliseconds, respectively. In the clustering model, services are clustered based on similarity, and each cluster includes a set of services. The clustering model is $50 \%$ faster than the model without clustering.

The search time for service discovery in the proposed model is based on 20 suppliers and 194 services equals to 3800 milliseconds. The proposed model is operating $75 \%$ faster than the uncluttered model for discovering services. Compared to the clustering model, it can search for query services for service discovery by $35 \%$ faster. In Figure 8 , the comparison of the proposed model with other models is shown on the basis of 15 suppliers and 150 services. The search time for the discovery of services in the proposed model is equal to 1,000 milliseconds. The proposed model with 15 suppliers and 150 services compared to no clustering model operates $90 \%$ faster. If the number of suppliers and services is lower, the search time for services to be discovered will be less because the number of comparisons will be less. On the other hand, if the number of suppliers and services is higher, users are more likely to discover their service.



Fig. 6. Ontology Model of Cloud Service Discovery on the basis of OWLViz Chart
TABLE I. EXAMPLES OF SPRALQL INSTRUCTIONS BASED ON THE PROPOSED MODEL

\begin{tabular}{|c|c|}
\hline $\begin{aligned} \text { SELECT ?SAAS WHERE }\{\text { ?Cloud_Service ?SAAS. } \\
\text { ?Cloud_Service rdfs:Ontlogy ?XML. } \\
\text { ?Cloud_Service rdfs:XML?SAAS.\} }\end{aligned}$ & (1) \\
\hline $\begin{array}{r}\text { SELECT ?PAAS WHERE \{?Cloud_Service ?PAAS. } \\
\text { ?Cloud_Service rdfs:Ontlogy ?RDF. } \\
\text { ?Cloud_Service rdfs:XML ?PAAS.\} }\end{array}$ & (2) \\
\hline $\begin{aligned} \text { SELECT ?IAAS WHERE }\{\text { ?Cloud_Service ?IAAS. } \\
\text { ?Cloud_Service rdfs:Ontlogy ?RDF. } \\
\text { ?Cloud_Service rdfs:XML ?IAAS.\} }\end{aligned}$ & (3) \\
\hline $\begin{array}{lrl}\text { SELECT } & \text { ?SAAS } & \text { ?PAAS ?IAAS WHERE }\{\text { ?Cloud_Service } \\
\text { ?SAAS. } & \\
& \text { ?Cloud_Service rdfs:Ontlogy ?XML. } \\
& \text { ?Cloud_Service rdfs:XML ?IAAS. } \\
\end{array}$ & (4) \\
\hline $\begin{array}{l}\text { SELECT ?IAAS (count(?Cloud_Service) as ? count) } \\
\text { WHERE \{?Cloud_Service ?IAAS.\} } \\
\text { GROUP BY ?IAAS }\end{array}$ & (5) \\
\hline 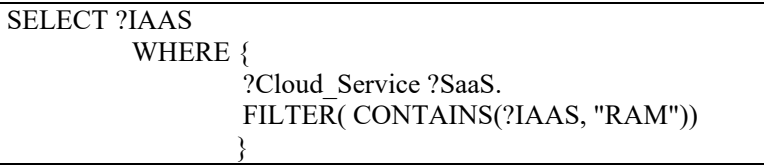 & (6) \\
\hline
\end{tabular}

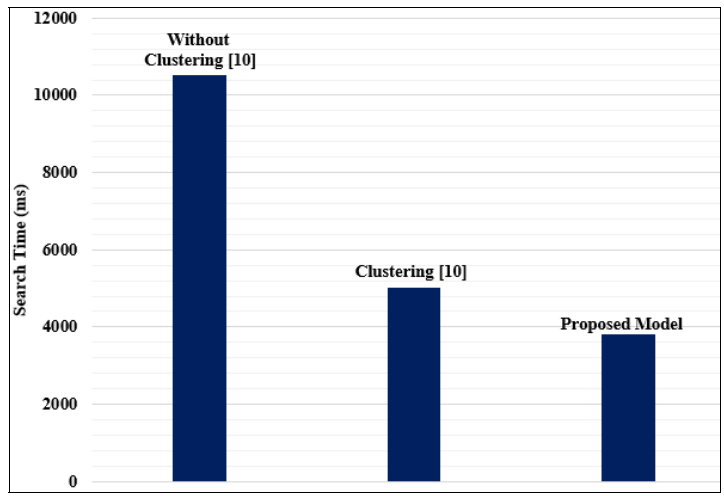

Fig. 7. Comparison of Proposed Model with other Models based on 20 Suppliers and 194 Services

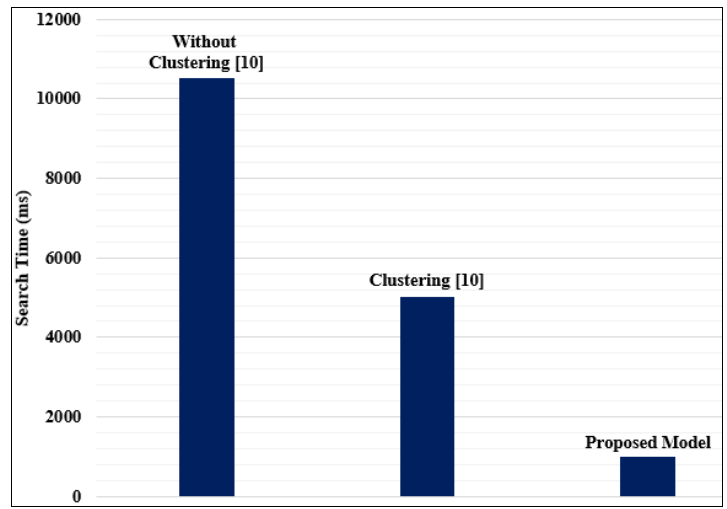

Fig. 8. Comparison of the proposed model with other models based on 15 suppliers and 150 services.

\section{CONCLUSION AND FUTURE WORKS}

Cloud computing is a model developed for easy, distributed, and comprehensive access to aggregate computing resources. In cloud computing, IT-based capabilities are 
presented as services that are accessible without the need for accurate knowledge of infrastructure technologies and the least managerial effort available. In cloud computing, cloud service discovery can satisfy user needs. Considering the relationships between cloud services based on ontology leads to the discovery of optimal cloud services. In addition to semantic relationships, standards such as XML, in the discovery of cloud services, also lead to enhanced service discovery. In this paper, we proposed a model for the discovery of cloud services based on ontology. We hope that in the future we will provide a system for discovering cloud services based on the ontology evolution and with applying techniques for the service interpretation, extraction and classification.

TABLE II. A COMPARISON OF SEARCH TIME FOR DISCOVERING SERVICES BASED ON THE NUMBER OF SERVICES AND PROVIDERS

\begin{tabular}{|c|c|c|c|}
\hline No & $\begin{array}{c}\text { Number of } \\
\text { suppliers }\end{array}$ & $\begin{array}{c}\text { Number of } \\
\text { service }\end{array}$ & $\begin{array}{c}\text { Search } \\
\text { time (MS) }\end{array}$ \\
\hline 1 & 15 & 150 & 1000 \\
\hline 2 & 15 & 100 & 1500 \\
\hline 3 & 15 & 120 & 1600 \\
\hline 4 & 20 & 100 & 1800 \\
\hline 5 & 20 & 150 & 2500 \\
\hline 6 & 20 & 194 & 3800 \\
\hline 7 & 25 & 100 & 2700 \\
\hline 8 & 25 & 120 & 3000 \\
\hline 9 & 30 & 150 & 3500 \\
\hline 10 & 40 & 150 & 4500 \\
\hline 11 & 45 & 100 & 6500 \\
\hline 12 & 50 & 100 & 7500 \\
\hline 13 & 50 & 150 & 8500 \\
\hline
\end{tabular}

\section{REFERENCES}

[1] P. Samimi, Y. Teimouri, M. Mukhtar, "A combinatorial double auction resource allocation model in cloud computing", Information Sciences, Vol. 357, pp. 201-216, 2016

[2] X. Ji, F. Zeng, M. Lin, "Data transmission strategies for resource monitoring in cloud computing platforms", Optik - International Journal for Light and Electron Optics, Vol. 127, No. 16, pp. 6726-6734, 2016

[3] W. Kong, Y. Lei, J. Ma, "Virtual machine resource scheduling algorithm for cloud computing based on auction mechanism", Optik - International Journal for Light and Electron Optics, Vol. 127, No. 12, pp. 5099-5104, 2016

[4] M. Rani, R. Nayak, O. P. Vyas, "An ontology-based adaptive personalized e-learning system, assisted by software agents on cloud storage", Knowledge-Based Systems, Vol. 90, pp. 33-48, 2015

[5] R. Guerfel, Z. Sbai, R. B. Ayed, "Towards a System for Cloud Service Discovery and Composition Based on Ontology", in: Lecture Notes in Computer Science, Vol. 9330, pp. 34-43, Springer, 2015

[6] A. Alfazi, T. H. Noor, Q. Z. Sheng, Y. Xu, "Towards OntologyEnhanced Cloud Services Discovery", in: Lecture Notes in Computer Science, Vol. 8933, pp. 616-629, Springer, 2014

[7] Y. M. Afify , I. F. Moawad, N. L. Badr, M. F. Tolba, "Cloud Services Discovery and Selection: Survey and New Semantic-Based System", in: Bio-inspiring Cyber Security and Cloud Services: Trends and Innovations, Vol. 70, pp. 449-477, Springer, 2014

[8] T. Labidi, A. Mtibaa, F. Gargouri, "Ontology-Based Context-Aware SLA Management for Cloud Computing, Model and Data Engineering", Lecture Notes in Computer Science, Vol. 8748, pp. 193-208, Springer, 2014
[9] M. Parhi, B. K. Pattanayak, M.R. Patra, “A Multi-agent-Based Framework for Cloud Service Description and Discovery Using Ontology", in: Intelligent Computing, Communication and Devices, Vol. 308, pp. 337-348, Springer, 2015

[10] T. Uchibayashi, B. Apduhan, N. Shiratori, "Towards a Cloud Ontology Clustering Mechanism to Enhance IaaS Service Discovery and Selection", International Conference on Computational Science and Its Applications, Part I, pp. 545-556, Springer, 2015

[11] M. Olaifa , S. Ojo, T. Zuva, "An Adaptive Multi Agent Service Discovery for Peer to Peer Cloud Services", in: Emerging Trends and Advanced Technologies for Computational Intelligence, Studies in Computational Intelligence, Vol. 647, pp. 147-163, Springer, 2016

[12] J. Shetty, D. A. D’Mello, “An XML based Data Representation Model to Discover Infrastructure Services", International Conference on Smart Technologies and Management for Computing, Communication, Controls, Energy and Materials, pp.119-125, 2015

[13] M. Rani, R. Nayak, O. P. Vyas, "An ontology-based adaptive personalized e-learning system, assisted by software agents on cloud storage", Knowledge-Based Systems, Vol. 90, pp. 33-48, 2015

[14] T. Han, K. M. Sim, "An Ontology-enhanced Cloud Service Discovery System", International MultiConference of Engineers and Computer Scientists, Vol. 1, pp. 1-6, 2010 